\title{
Legionella Pneumonia Immediately after Recovery from COVID-19
}

\author{
Daisuke Jingu ${ }^{1}$, Hiroshi Takahashi ${ }^{1}$, Satoshi Ubukata ${ }^{1}$, Hiroshi Watanabe ${ }^{1}$, and Akira \\ Horii $^{1}$ \\ ${ }^{1}$ Saka General Hospital
}

February 8, 2022

\begin{abstract}
We experienced a patient with Legionella Pneumonia immediately after discharge from COVID-19. It is very important to accurately detect pathogens of infectious diseases particularly in the time of COVID-19 pandemic.
\end{abstract}

Title

Legionella Pneumonia Immediately after Recovery from COVID-19

Authors

Daisuke Jingu $^{1}$, Hiroshi Takahashi ${ }^{1}$, Satoshi Ubukata ${ }^{1}$, Hiroshi Watanabe ${ }^{1}$ and Akira Horii ${ }^{2}$

Departments of ${ }^{1}$ Respiratory Medicine and ${ }^{2}$ Internal Medicine, Saka General Hospital, 16-5 Nishiki-cho, Shiogama, Miyagi, 9858506, Japan

\section{Correspondence}

Daisuke Jingu, Department of Respiratory Medicine, Saka General Hospital, 16-5 Nishiki-cho, Shiogama, Miyagi, 985-8506, Japan

Email: d.jinguuu@gmail.com

\section{Running head}

Legionella Pneumonia Immediately after Recovery from COVID-19

\section{Abstract}

We experienced a patient with Legionella Pneumonia immediately after discharge from COVID-19. It is very important to accurately detect pathogens of infectious diseases particularly in the time of COVID-19 pandemic.

\section{Key Clinical Message}

We experienced a patient with Legionella Pneumonia immediately after discharge from COVID-19. It is very important to accurately detect pathogens of infectious diseases particularly in the time of COVID-19 pandemic.

\section{KEYWORDS}

COVID-19, SARS-CoV-2, Legionella pneumonia, Legionella pneumophila, serogroup 5

\section{CLINICAL IMAGE}


An 83-year-old woman, living alone, visited our emergency department with consciousness disorder, high fever $\left(39.3^{\circ} \mathrm{C}\right)$, and hypoxemia. She had been discharged only 5 days earlier after 10 days of hospitalization for COVID-19. Examination data revealed high inflammatory responses with acute kidney injury. Chest $\mathrm{X}$-ray and $\mathrm{CT}$ demonstrated features of severe pneumonia, mainly in the right lobe (Figure 1), which are obviously distinct from those of COVID-19 (Figure 2). The SARS-CoV-2 antigen was negative, but urinary Legionellaantigen was positive; Legionella pneumonia was diagnosed. Her sputum disclosed Legionella pneumophila (serogroup 5). Treatment with antibiotics was successful. The patient gradually recovered and was transferred to a long-term hospital on day 68. In addition, Legionella pneumophila was also detected in her daily reused bathtub water. This very common lifestyle in Japan can be a hotbed of Legionella infection in this case. Furthermore, increasing concerns on the Legionnaires' disease are warned in association with release or relaxation from lockdown after the burning down of COVID-19 pandemic. ${ }^{1,2}$ Infectious diseases such that caused by Legionella should also be considered for patients after discharge.

\section{CONSENT STATEMENT}

Written informed consent was obtained from the patient to publish this report in accordance with the journal's patient consent policy, and the study was approved by the Ethics Committee in Saka General Hospital (No. 21-9-21).

\section{DATA AVAILABILITY}

No data available in this case report.

\section{ACKNOWLEDGMENTS}

We are grateful to Barbara Lee Smith Pierce (Retired Professor, University of Maryland University College) for her editorial work in the preparation of the manuscript.

\section{CONFLICT OF INTEREST}

None declared.

\section{AUTHOR CONTRIBUTIONS}

DJ, HT, SU and HW substantially contributed to the diagnosis and clinical care of the patient. DJ wrote the draft of the manuscript, and DJ and AH critically revised it for important intellectual content.

\section{FUNDING INFORMATION}

None.

\section{ORCID}

Daisuke Jinguhttps://orcid.org/0000-0002-0153-3912

Akira Horiihttps://orcid.org/0000-0002-3967-3291

\section{REFFERENCES}

1. Cassell K, Davis JL, Berkelman R . Legionnaires' disease in the time of COVID-19. Pneumonia. 2021;13:2. doi: 10.1186/s41479-020-00080-5.

2. Palazolo C, Maffongelli M, D'Abramo A, et al. Legionellapneumonia: increased risk after COVID19 lockdown? Italy, May to June 2020. Euro Surveill. 2020;25:2001372. doi: 10.2807/15607917.ES.2020.25.30.2001372.

\section{Legend to Figures}

FIGURE 1. On admission with Legionella pneumonia. Results of chest X-ray and CT scan demonstrated acute inflammatory changes, mainly in the right lobe. 
FIGURE 2. On the previous admission with COVID-19 infection. Results of chest X-ray and CT scan showed ground-glass opacities bilaterally with peribronchovascular distribution.

Figure 1
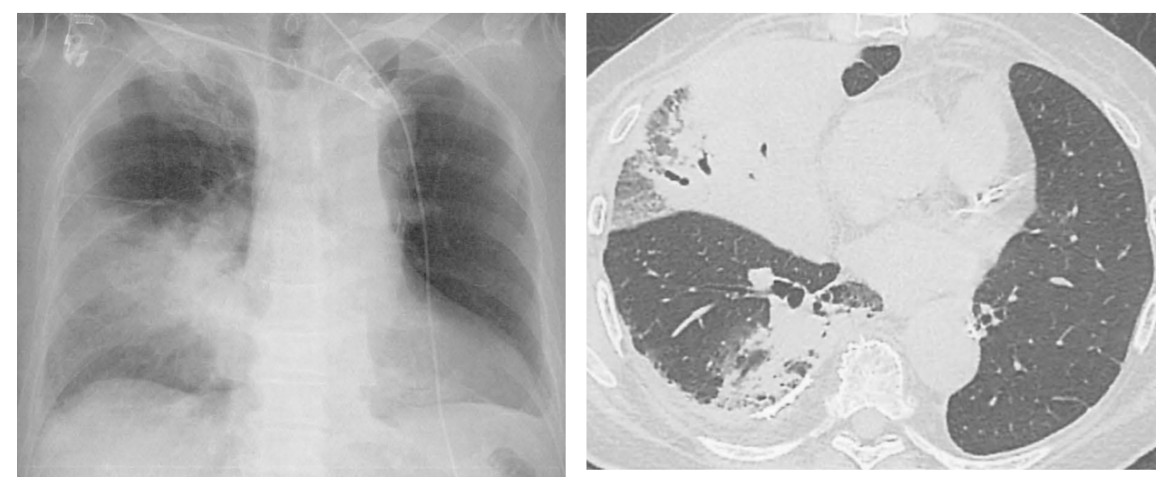

Figure 2
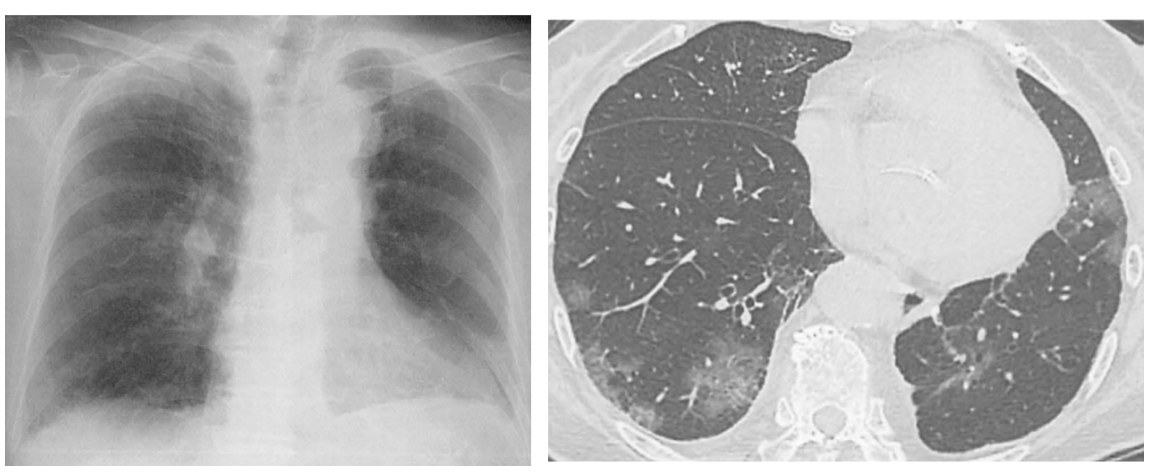